Mini-Review

\title{
Suicide Gene Therapy to Increase the Safety of Chimeric Antigen Recep- tor-Redirected T Lymphocytes
}

\author{
Monica Casucci, Attilio Bondanza ${ }^{凶}$ \\ Experimental Hematology and Bone Marrow Transplantation Unit, San Raffaele Hospital, Milano, ITALY
}

Corresponding author: Attilio Bondanza, MD PhD, Experimental Ematology and Bone Marrow Transplantation Unit, Division of Regenerative Medicine, Stem Cell and Gene Therapy, S. Raffaele Scientific Institute, Via Olgettina, 5820132 Milano (ITALY). Tel.+39-02-2643-5612; Fax +39-02-2643-4786; Email: bondanza.attilio@hsr.it

(C) Ivyspring International Publisher. This is an open-access article distributed under the terms of the Creative Commons License (http://creativecommons.org/ licenses/by-nc-nd/3.0/). Reproduction is permitted for personal, noncommercial use, provided that the article is in whole, unmodified, and properly cited.

Received: 2011.06.10; Accepted: 2011.06.16; Published: 2011.07.01

\begin{abstract}
Chimeric antigen receptors (CARs) are generated by fusing the antigen-binding motif of a monoclonal antibody $(\mathrm{mAb})$ with the signal transduction machinery of the T-cell receptor (TCR). The genetic modification of T lymphocytes with chimeric receptors specific for tumor-associated antigens (TAAs) allows for the redirection towards tumor cells. Clinical experience with CAR-redirected T cells suggests that antitumor efficacy associates with some degree of toxicity, especially when TAA expression is shared with healthy tissues. This situation closely resembles the case of allogeneic hematopoietic stem cell transplantation (HSCT), wherein allorecognition causes both the graft-versus-leukemia (GVL) effect and graft-versus-host disease (GVHD). Suicide gene therapy, i.e. the genetic induction of a conditional suicide phenotype into donor T cells, enables dissociating the GVL effect from GVHD. Applying suicide gene modification to CAR-redirected T cells may therefore greatly increase their safety profile and facilitate their clinical development.
\end{abstract}

Key words: Suicide Gene Therapy, Chimeric antigen receptors

\section{Suicide gene therapy and HSCT}

In HSCT, the natural alloreactivity of donor $\mathrm{T}$ lymphocytes play a major role in the eradication of the underlying malignant disease in the so-called GVL effect ${ }^{1}$. However, the majority of alloantigens are not only expressed by leukemic cells, but also by normal cells. The GVL effect is therefore often accompanied by an alloimmune attack against healthy tissues, whose manifestations are known as GVHD. GVHD is a severe disease that is often lethal. GvHD prevention is accomplished through either T-cell depletion of the graft or post-transplant immune suppression. Although effective in abating GVHD incidence and severity, both strategies unfortunately associate with a significant reduction in the GVL effect, thus jeopardizing the overall efficacy of HSCT.
Alternatively to avoiding alloreactivity tout-court, suicide gene therapy proposes to exploit the GVL effect, while having the possibility to switch off GVHD at will ${ }^{2}$. This goal is achieved through the ex vivo genetic modification of $\mathrm{T}$ lymphocytes with a suicide gene, i.e. a gene encoding for a factor able to convert at a cellular level a non-toxic prodrug into a toxic compound. During GVHD, the administration of the prodrug selectively eliminates suicide gene-modified $\mathrm{T}$ cells without interfering with the process of immune reconstitution operated by unmodified $\mathrm{T}$ cells. Suicide gene therapy in the context of HSCT has been pioneered by our group in the mid-90s with the use of the Herpes simplex thymidine kinase (Hsv-tk)/ganciclovir (GCV) suicide system ${ }^{3}$. 
Since then, more than 100 patients have been treated worldwide with Hsv-tk-modified $\mathrm{T}$ cells, demonstrating that the strategy is safe and efficacious. Interestingly, data from phase I/II clinical trials have confirmed earlier studies in humanized mouse models, which predicted that having GVHD and the GVL effect different kinetics and effector mechanisms, the therapeutic benefit of HSCT wouldn't be put at risk by the successful activation of the suicide gene machinery ${ }^{4}$. Moreover, recent improvements in the protocols for genetic modification of primary $\mathrm{T}$ cells with retroviral vectors are expected to further increase the effectiveness of suicide gene therapy with Hsv-tk-modified T cells ${ }^{5-7}$.

Despite being the first-in-class suicide gene and having clearly demonstrated safety and efficacy, HSV-tk has some limitations ${ }^{8}$. The first, and most obvious, is that being of viral origin HSV-tk is immunogenic and therefore may cause the rejection of modified $\mathrm{T}$ lymphocytes by the immune response of the host. The second problem of HSV-tk is its clinical incompatibility with the use of GCV for antiviral purposes. HSCT patients often reactivate cytomegalovirus (CMV), which if treated with GCV results in the unwanted elimination of modified $\mathrm{T}$ cells. The third drawback of HSV-tk derives form its mechanism of suicide action. HSV-tk-modified $\mathrm{T}$ cells acquire the ability to phosphorilate GCV, which once incorporated in the genome causes apoptosis upon cell-cycle entry. Therefore, only modified cells that do proliferate are killed by GCV.

In order to solve the limitations of HSV-tk, various alternative suicide gene systems have been proposed (see Table 1). The genetic modification of $\mathrm{T}$ lymphocytes with the B-cell molecule $C D 20^{9}$, for example, allows their elimination upon administration of mAb Rituximab. The major advantage of $C D 20$ as a suicide gene is that being of human origin it is not expected to be immunogenic. Moreover, given the mechanism of action of Rituximab, CD20-modified T cells are possibly eliminated regardless of their proliferation status. A possible pitfall of the CD20/Rituximab suicide gene system, however, relates to the characteristics of the prodrug, given that the biodistribution of a mAb is clearly sub-optimal compared to a small molecule such as GCV. In addition, the administration of Rituximab for the activation of the suicide gene machinery will predictably cause the unwanted depletion of normal B cells. Other non-immunogenic alternatives to HSV-tk include suicide genes based on the chemical inducers of dimerization $(\mathrm{CID})^{10}$. According to this technology, a proapototic molecule is adapted to encompass one or more binding sites for a CID, which once reaching its target(s) causes their oligomerization with ensuing activation of the apoptotic pathway. In this way, different apoptotic pathways have been employed as suicide systems, including the death receptor Fas ${ }^{11}$ and the enzyme caspase 912 . Beside a very low risk for immunogenicity, these suicide genes share the advantages of non cell-cycle dependency, full clinical compatibility and optimal biodistribution, as CID are small molecule exquisitely designed for suicide purposes. As a general consideration, however, it has to be underlined that at the current state of the art, only HSV-tk has been applied in humans, and that the theoretical advantages of other suicide genes await clinical validation.

Table I. Biological characteristics of the different suicide genes proposed for use in hematopoietic stem cell transplantation

\begin{tabular}{lccc}
\hline & HSV-tk & CD20 & $\Delta$ Fas $/ i$ Casp9 ฯฯ \\
\hline Immunogenicity & + & - & - \\
Clinical compatibility & - & - & + \\
Cell-cycle dependency & + & - & - \\
\hline
\end{tabular}

I Clinical incompatibility of $C D 20$ as a suicide gene refers to the unwanted elimination of normal B cells upon administration of Rituximab for suicide purposes.

ฯ $\Delta F a s$ is derived from the truncation of the intracellular domain of the proapototic molecule Fas and its fusion to CID-binding domains. By analogy, inducible caspase 9 (iCasp 9 ) is derived from the fusion of caspase 9 to CID-binding domains.

\section{Suicide gene therapy with CAR-redirected T cells}

Adoptive T-cell therapy with CAR-redirected T lymphocytes is at the frontier of modern cancer treatment ${ }^{13}$. The possibility to redirect $\mathrm{T}$ cells towards virtually any surface TAA by means of gene therapy, in fact, opens for the exploitation of the extraordinaire therapeutic potential of $\mathrm{T}$ cells for an increasing number of patients suffering from cancer. Firstly, pioneered by Zelig Eshhar ${ }^{14}$, the concept of the genetic redirection of $\mathrm{T}$ cells by means of a chimeric receptor linking the antigen-binding moiety of a $\mathrm{mAb}$ in the form of a single-chain fragment variable $(\mathrm{scFv})$ region to the signal transduction machinery of $\mathrm{T}$ cells is now being translated into the clinic at an incredible pace. Currently, there are a number of active and recruiting phase I/II clinical trials aiming at the demonstration of the safety and the efficacy of CARs both in the US and in the European Union ${ }^{13}$. The majority of clinical trials are focused on lineage marker-specific CARs, that is CARs specific for molecules selectively expressed 
by the malignant counterpart of normal blood cells. The most notable examples are CD19-specific CARs, which are now being investigated for the treatment of different B-cell tumors such as chronic lymphocytic leukemia (CLL), acute lymphoid leukemia and lymphoma. Hematological malignancies, however, are not the only tumors targeted by CARs. More recently a number of novel oncoantigen-specific CARs, that is CARs specific for antigens whose expression is somewhat linked to the malignant phenotype, have been described and have entered the clinical arena for some types of solid tumors.

The appeal of CARs for cancer treatment is multifaceted (see Table 2). CARs share with $\mathrm{mAb}$ the profit of targeting a surface TAA regardless of its nature, protein, carbohydrate or lipid, and, most importantly, of antigen processing and presentation in the context of MHC. Differently from TCR-mediated recognition, CAR-mediated recognition is therefore insensitive to tumor escape mechanisms such as antigen processing defects and/or down regulation of MHC expression. A logical consequence of MHC-independent recognition is that CARs are widely applicable no matter the HLA repertoire of the patients. Apart from this, CAR-redirection benefits from all the advantages of tumor targeting with $\mathrm{T}$ cells, including optimal biodistribution and the ability of persist long term, properties that fall short with mAbs. Moreover, $\mathrm{T}$ cells can be complemented with genes that enable new properties, such as resistance to immunosuppressive circuits and, crucially, a conditional suicidal phenotype as in the case of HSV-tk and other suicide genes.

Table 2. Schematic comparison of the advantages/ disadvantages of $m A b$, chimeric antigen receptor (CAR)and clonal TCR-redirected T cells for cancer therapy

\begin{tabular}{lccc}
\hline & $\mathrm{mAb}$ & $\mathrm{CAR}$ & $\mathrm{TCR}$ \\
\hline MHC restriction & - & - & + \\
Ag processing & - & - & + \\
Persistence & - & + & + \\
Biodistribution & - & + & + \\
Suicide switch & - & + & + \\
\hline
\end{tabular}

The first clinical experience with CAR-redirected $\mathrm{T}$ cells was rather disappointing, showing very poor in vivo persistence and efficacy. Such a behavior was soon linked to the lack of costimulation provided by early, or first generation (1G) CARs. Accordingly, the provision of costimulation in trans via the genetic modification of T-cell lines specific for latent viruses such as Epstein Barr virus proved to ameliorate in vivo persistence and to cause impressive tumor regressions ${ }^{15}$. Alternatively, the progressive addition within the CAR backbone of a CD28 costimulatory endodomain (2G CAR) has recently been demonstrated to dramatically increase in vivo persistence ${ }^{16}$. On the same line, the implementation of a second costimulatory endodomain, either from OX40 or 4-1BB, has also been proposed (3G CAR) Unfortunately, along with hints of clinical efficacy, 2G and 3G CARs have also born important safety concerns, as two serious adverse events (SAEs) determining the fatal outcome of the treated patients were reported in two distinct clinical trials. The first occurred in a CLL patient treated with $T$ cells redirected with a 2 G CAR specific for CD19 at the Sloan-Kettering Cancer Center in New York ${ }^{17}$, while the second occurred in a patient with colorectal cancer treated $\mathrm{T}$ cells redirected with a $2 \mathrm{G}$ CAR specific for CD19 at the National Institute of Health in Bethesda ${ }^{18}$. In the first case, the SAE was later recognized as a tumor-lysis syndrome, and therefore not primarily related to the biology of CAR-redirected $\mathrm{T}$ cells. In the second case, however, the SAE was likely to be directly caused by excessive cytokine release upon on-target recognition, which possibly included also lung epithelia expressing low levels of HER2/neu.

The association between clinical efficacy and toxicity when applying CAR-redirected $\mathrm{T}$ cells for cancer treatment much resembles the case of HSCT, wherein the GVL effect and GVHD are tightly linked phenomenons. Since suicide gene therapy for HSCT has demonstrated the potential to operate a meaningful dissociation between the GVL effect and GVHD, it is reasonable to think that the implementation of a suicide gene in CAR-redirected $\mathrm{T}$ cells may help mitigating their risks, while preserving their therapeutic effect. The use of a suicide gene may be particularly appropriate when targeting oncoantigens whose expression is leaky and therefore present, albeit at lower levels, on healthy tissues. Due to previous experience with CAR-redirected T cells, it is unlikely that suicide genes would work as a universal surrogate for safety. For example, by no means, suicide genes may substitute from a correct clinical development plan, including the cautious choice of the starting dose when applying a specific CAR for the first time in humans or a careful intra- or inter-patient dose escalation to avoid early or idiosynchrasic toxicities ${ }^{19}$. None withstanding this, suicide genes may prove greatly helpful in managing intermediate and late toxicities not only during the different phases of clinical development, but especially, and hopefully, in the case CAR-redirected T cells would finally translate into mainstream medicine. 


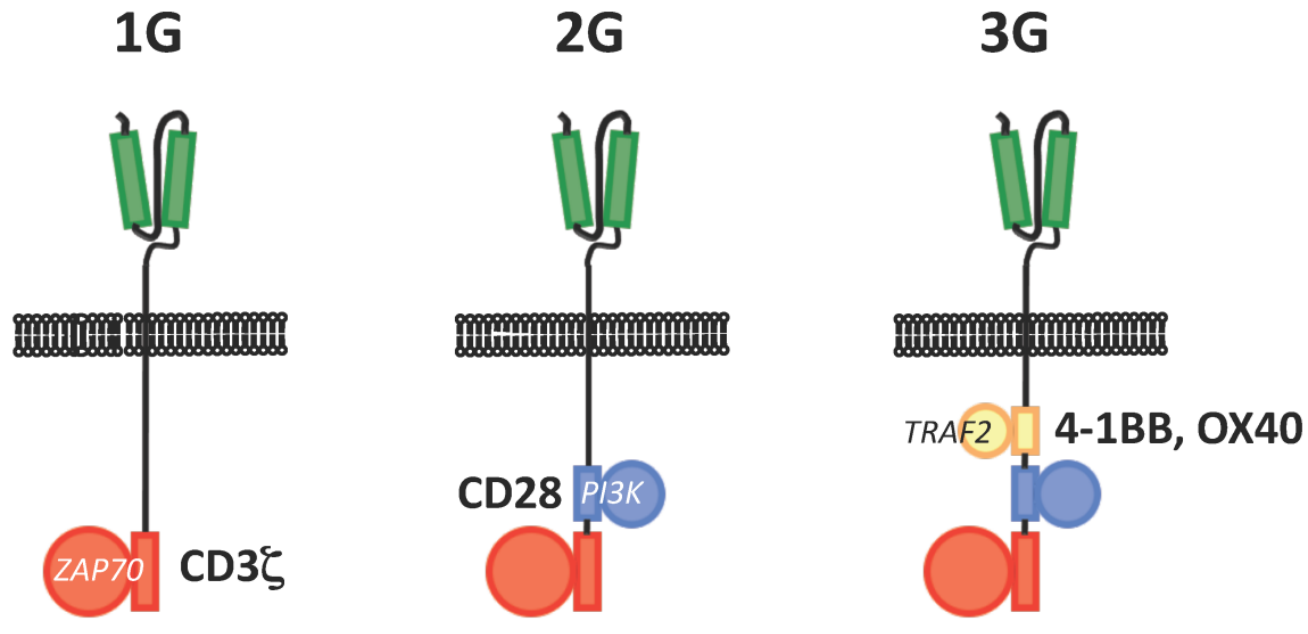

\section{Cytotoxicity}

Proliferation/cytokine production

Survival

Figure I. Schematic representation of the different generations of CARs (IG, first generation, 2G, second generation, 3G, third generation). The scFv is highlighted in green, while the different component of TCR signal transduction machinery are highlighted in red (CD3 $\zeta$ chain/ZAP70), blue (CD28/PI3K) and yellow (4-IBB or OX40/TRAF).

\section{Conflict of Interest}

The authors have declared that no conflict of interest exists.

\section{References}

1. Appelbaum FR. Haematopoietic cell transplantation as immunotherapy. Nature. 2001;411(6835):385-389.

2. Bondanza A, Ciceri F, Bonini C. Application of donor lymphocytes expressing a suicide gene for early GVL induction and later control of GVH reactions after bone-marrow transplantation. Methods Mol Med. 2005;109:475-486.

3. Bonini C, Ferrari G, Verzeletti S, et al. HSV-TK gene transfer into donor lymphocytes for control of allogeneic graft-versus-leukemia. Science. 1997;276(5319):1719-1724.

4. Ciceri F, Bonini C, Marktel S, et al. Antitumor effects of HSV-TK-engineered donor lymphocytes after allogeneic stem-cell transplantation. Blood. 2007;109(11):4698-4707.

5. Bondanza A, Valtolina V, Magnani Z, et al. Suicide gene therapy of graft-versus-host disease induced by central memory human T lymphocytes. Blood. 2006;107(5):1828-1836.

6. Kaneko S, Mastaglio S, Bondanza A, et al. IL-7 and IL-15 allow the generation of suicide gene-modified alloreactive self-renewing central memory human T lymphocytes. Blood. 2009;113(5):1006-1015.

7. Bondanza A, Hambach L, Aghai Z, et al. IL-7 receptor expression identifies suicide gene-modified allospecific CD8+ T cells capable of self-renewal and differentiation into antileukemia effectors. Blood. 2011; Epub.

8. Lupo-Stanghellini MT, Provasi E, Bondanza A, Ciceri F, Bordignon $\mathrm{C}$, Bonini $\mathrm{C}$. Clinical impact of suicide gene therapy in allogeneic hematopoietic stem cell transplantation. Hum Gene Ther. 2010;21(3):241-250.

9. Introna M, Barbui AM, Bambacioni F, et al. Genetic modification of human T cells with CD20: a strategy to purify and lyse transduced cells with anti-CD20 antibodies. Hum Gene Ther. 2000;11(4):611-620.

10. Rollins CT, Rivera VM, Woolfson DN, et al. A ligand-reversible dimerization system for controlling protein-protein interactions. Proc Natl Acad Sci U S A. 2000;97(13):7096-7101.

11. Thomis DC, Marktel S, Bonini C, et al. A Fas-based suicide switch in human $\mathrm{T}$ cells for the treatment of graft-versus-host disease. Blood. 2001;97(5):1249-1257.

12. Straathof KC, Pule MA, Yotnda P, et al. An inducible caspase 9 safety switch for T-cell therapy. Blood. 2005;105(11):4247-4254.

13. Jena B, Dotti G, Cooper LJ. Redirecting T-cell specificity by introducing a tumor-specific chimeric antigen receptor. Blood. 2011;116(7):1035-1044.

14. Gross G, Waks T, Eshhar Z. Expression of immunoglobulin-T-cell receptor chimeric molecules as functional receptors with antibody-type specificity. Proc Natl Acad Sci U S A. 1989;86(24):10024-10028.

15. Pule MA, Savoldo B, Myers GD, et al. Virus-specific T cells engineered to coexpress tumor-specific receptors: persistence and antitumor activity in individuals with neuroblastoma. Nat Med. 2008;14(11):1264-1270.

16. Savoldo B, Ramos CA, Liu E, et al. CD28 costimulation improves expansion and persistence of chimeric antigen receptor-modified $\mathrm{T}$ cells in lymphoma patients. J Clin Invest. 2011;121(5):1822-1826.

17. Brentjens R, Yeh R, Bernal Y, Riviere I, Sadelain M. Treatment of chronic lymphocytic leukemia with genetically targeted autologous T cells: case report of an unforeseen adverse event in a phase I clinical trial. Mol Ther. 2010;18(4):666-668. 
18. Morgan RA, Yang JC, Kitano M, Dudley ME, Laurencot CM, Rosenberg SA. Case report of a serious adverse event following the administration of $\mathrm{T}$ cells transduced with a chimeric antigen receptor recognizing ERBB2. Mol Ther. 2010;18(4):843-851.

19. Ertl HC, Zaia J, Rosenberg SA, et al. Considerations for the Clinical Application of Chimeric Antigen Receptor T Cells: Observations from a Recombinant DNA Advisory Committee Symposium Held June 15, 2010. Cancer Res. 2011;71(9):3175-3181. 